\title{
Chebyshev expansion for the component functions of the almost-Mathieu operator
}

\author{
Jesús C. Abderramán Marrero \\ Applied Math Dpt. - Faculty of Computer Science - U.P.M.(Technological University of Madrid) Campus Montegancedo \\ 28660 - Boadilla Madrid. Spain.
}

\begin{abstract}
The component functions $\left\{\Psi_{n}(\epsilon)\right\}\left(n \in \mathcal{Z}^{+}\right)$from difference Schrödinger operators, can be formulated in a second order linear difference equation. Then the Harper equation, associated to almost-Mathieu operator, is a prototypical example. Its spectral behavior is amazing. Here, due the cosine coefficient in Harper equation, the component functions are expanded in a Chebyshev series of first kind, $T_{n}(\cos 2 \pi \theta)$. It permits us a particular method for the $\theta$ variable separation. Thus, component functions can be expressed as an inner product, $\Psi_{n}(\epsilon, \lambda, \theta)=\vec{T}_{\left[\frac{n(n-1)}{2}\right]}(\cos 2 \pi \theta) \cdot \vec{A}_{\left[\frac{n(n-1)}{2}\right]}(\epsilon, \lambda)$. A matrix block transference method is applied for the calculation of the vector $\vec{A}_{\left[\frac{n(n-1)}{2}\right]}(\epsilon, \lambda)$. When $\theta$ is integer, $\Psi_{n}(\epsilon)$ is the sum of component from $\vec{A}_{\left[\frac{n(n-1)}{2}\right]}$. The complete family of Chebyshev Polynomials can be generated, with fit initial conditions. The continuous spectrum is one band with Lebesgue measure equal to 4 . When $\theta$ is not integer the inner product $\Psi_{n}$ can be seen as a perturbation of vector $\vec{T}_{\left[\frac{n(n-1)}{2}\right]}$ on the sum of components from the vector $\vec{A}_{\left[\frac{n(n-1)}{2}\right]}$. When $\theta=\frac{p}{q}$, with $p$ and $q$ coprime, periodic perturbation appears, the connected band from the integer case degenerates in q sub-bands. When $\theta$ is irrational, ergodic perturbation produces that one band spectrum from integer case degenerates to a Cantor set. Lebesgue measure is $L_{\sigma}=4(1-|\lambda|), 0<|\lambda| \leq 1$. In this situation, the series solution becomes critical.
\end{abstract}

\section{Chebyshev expansion of the component functions.}

The Almost-Mathieu operator appears in some approximated quantum models of energy spectra, This operator can be formulated via a second order linear difference equation, known as Harper equation:

$$
\Psi_{n+1}(\epsilon)=(\epsilon-2 \lambda \cos (n 2 \pi \theta+\nu)) \Psi_{n}(\epsilon)-\Psi_{n-1}(\epsilon) .
$$

The family of component functions $\left\{\Psi_{n}(\epsilon, \lambda, \theta, \nu)\right\}$, depends of $\epsilon$, the energy, as primary parameter, and the other parameters related with the particular characteristics of the system in study. When $\theta$ is irrational, ergodic case, many work has been generated, focussed on spectrum analysis.

The form of (1) suggests a solution in series of Chebyshev polynomials of first kind. Also, when $n \rightarrow \infty$, this series type converges in $\mathcal{L}_{2}, \quad$ Product properties of Chebyshev polynomials of first kind $T_{n}(\omega)$ are used. Here, without loss of generality, $\nu=0$. Indeed, when $\theta$ is irrational the spectrum does not depend on $\nu$. In other situations, variations on $\nu$ only produces shifts in all spectrum bands. This series must agree with Eq. (1). Thus, for $n$ finite, the series is truncated.

$$
\Psi_{n}(\epsilon)=\sum_{k=0}^{\left[\frac{n(n-1)}{2}\right]} a_{k}^{(n)}(\epsilon, \lambda) T_{k}(\omega) .
$$

With $[x]$ the integer component of $x$, and $\omega=2 \pi \theta$. Eq. (2) is introduced in (1) and coefficients from $T_{n}(\omega)$ with equal $n$ are matched. A recurrent expression for the coefficients $a_{k}^{(n)}(\epsilon, \lambda)$ are obtained. The compact form is:

$$
\begin{aligned}
a_{k}^{(n+1)}= & -\lambda a_{k-n}^{(n)} \sigma(n-1)+\epsilon a_{k}^{(n)}\left(1-\sigma\left(\frac{(n)(n-1)}{2}\right)\right)-\lambda a_{n-k}^{(n)}(1-\sigma(n)) \\
& -\lambda a_{k+n}^{(n)}\left(1-\delta_{k, 0}-\sigma\left(\frac{(n)(n-3)}{2}\right)\right)-a_{k}^{(n-1)}\left(1-\sigma\left(\frac{(n-1)(n-2)}{2}\right)\right) .
\end{aligned}
$$

With $\sigma(k)$ the Heaviside step function and $\delta_{k, 0}$ the Kronecker delta function, $0 \leq k \leq \frac{(n+1)(n)}{2}$.

\subsection{Variable separation and inner product.}

The parameters are separated. The coefficients of the series depend from $\epsilon$ and $\lambda$, the Chebyshev Polynomials $T_{n}(\omega)$ depend from $\theta$. Eq. (2) can be seen as an inner product, $\Psi_{n}(\epsilon, \lambda, \theta)=\vec{T}_{\left[\frac{n(n-1)}{2}\right]}^{T}(\omega) \cdot \vec{A}_{\left[\frac{n(n-1)}{2}\right]}(\epsilon, \lambda)$. The vector $\vec{T}_{\left[\frac{n(n-1)}{2}\right]}$, with components $t_{i}=\cos (2 i \pi \theta), i=0,1, \ldots\left[\frac{n(n-1)}{2}\right]$, the vector $\vec{A}_{\left[\frac{n(n-1)}{2}\right]}$ is generated via the recursion from (3). 


\subsection{Transference Block Matrix for the vector $\vec{A}_{\left[\frac{n(n-1)}{2}\right]}$.}

Matrix transference method can be used to find a suitable linear recursion map This recursion permits us the achievement of $\vec{A}_{\left[\frac{n(n-1)}{2}\right]}$. The simplest recursion is the linear first order recursion one. With this purpose, it is necessary to work with a double vector $\overrightarrow{\hat{A}}$, which contains both vectors, $\vec{A}_{\left[\frac{n(n-1)}{2}\right]}$ and $\vec{A}_{\left[\frac{(n-1)(n-2)}{2}\right]}$. $\overrightarrow{\hat{A}}_{n}^{T}=\left(\vec{A}_{\left[\frac{n(n-1)}{2}\right]}^{T}, \vec{A}_{\left[\frac{(n-1)(n-2)}{2}\right]}^{T}\right)$.

The vector recursion, for $n \geq 2$, with initial vector $\overrightarrow{\hat{A}}_{1}=\left(\begin{array}{l}\Psi_{1} \\ \Psi_{0}\end{array}\right)$, is $\overrightarrow{\hat{A}}_{n}=\mathbf{M}_{n, n-1} \overrightarrow{\hat{A}}_{n-1}$, with the block matrix:

$$
\mathbf{M}_{n, n-1}=\left(\begin{array}{c|c|c}
\epsilon \mathbf{I}_{n-1}-\lambda \mathbf{L}_{n-1} & -\lambda \mathbf{I}_{\left[\frac{(n-1)(n-4)}{2}\right]+1} & -\mathbf{I}_{\left[\frac{(n-2)(n-3)}{2}\right]+1} \\
\hline-\lambda\left(\mathbf{I}_{1}+\mathbf{I}_{n-1}\right) & \epsilon \mathbf{I}_{\left[\frac{(n-1)(n-4)}{2}\right]+1} & \mathbf{0} \\
\hline 0 & -\lambda\left(\mathbf{I}_{\left[\frac{(n-1)(n-4)}{2}\right]+1}\right. & \mathbf{0}
\end{array}\right) .
$$

Rows and columns are labelled from 0 to $n-1$. The $\mathbf{I}_{n}$ matrix is the identity matrix of order $n$. $\mathbf{L}_{n}$ is the matrix with component $l_{i, j}=\delta_{n, i+j}$, Kronecker delta, with $0 \leq i, j \leq n-1$. The $\mathbf{L}_{n}$ matrix mixes coefficients and it complicates the recursion. For example, the block matrix $\mathbf{M}_{3,2}$ is:

$$
\left(\begin{array}{cc|c}
\epsilon & 0 & -1 \\
0 & \epsilon-\lambda & 0 \\
\hline-2 \lambda & 0 & 0 \\
0 & -\lambda & 0 \\
\hline 1 & 0 & 0 \\
0 & 1 & 0
\end{array}\right)
$$

When $\theta$ is integer, $\vec{T}_{\left[\frac{n(n-1)}{2}\right]}=\overrightarrow{1}_{\left[\frac{n(n-1)}{2}\right]}$, with $\frac{n(n-1)}{2}+1$ vector components $t_{i}=1$. The $\Psi_{n}$ function is equal to the sum of component from the vector $\vec{A}_{\left[\frac{n(n-1)}{2}\right]}$. The complete family of Chebyshev Polynomials can be generated, with fit initial conditions. For example, if $\Psi_{0}=1$, and $\Psi_{1}=2\left(\frac{\epsilon}{2}-\lambda \cos (\nu)\right)$, then, $\Psi_{n}=U_{n}\left(\frac{\epsilon}{2}-\lambda \cos (\nu)\right)$, Chebyshev Polynomials of second kind, in $\epsilon$ variable. Observe that, in this problem, this is the unique family of monic polynomials, [3], that converges in $\mathcal{L}_{2}$-morm

\section{Continuous spectrum and the vector $\vec{T}_{\left[\frac{n(n-1)}{2}\right]^{\circ}}$.}

When $\theta$ is integer, Eq. (1) is trivial. The continuous spectrum appears in the band $[-2+2 \lambda \cos (\nu), 2+2 \lambda \cos (\nu)]$, located into the compact $[-4,4]$, with Lebesgue measure equal to 4 .

When $\theta$ is not integer, the inner product $\Psi_{n}$ can be seen as a perturbation of vector $\vec{T}_{\left[\frac{n(n-1)}{2}\right]}$ on the sum of components from vector $\vec{A}_{\left[\frac{n(n-1)}{2}\right]}$. For $\theta=\frac{p}{q}$, with $p$ and $q$ coprime, $\vec{T}_{\left[\frac{n(n-1)}{2}\right]}$ has q-periodic components, and one periodic perturbation appears. Now, the connected spectrum band, from the $\theta$ integer case, degenerates in $q$ sub-bands.

If $\theta$ is irrational, the component from the $\vec{T}_{\left[\frac{n(n-1)}{2}\right]}$ are quasi-periodic. The perturbation on the sum becomes ergodic. This produces that the continuous spectrum, of integer $\theta$ case, degenerates to a Cantor set, with Lebesgue measure $L_{\sigma}=4(1-|\lambda|)$, $0<|\lambda| \leq 1$, The solutions from Eq.(2) in these Cantor sets are critical. A rigorous argument for this situation is an open line.

\section{References}

D.F. Hofstadter, Energy levels and waves functions of Bloch electrons in rational and irrational magnetic fields. Physical Review B, 14(6), 455-460 (1976).

J. Puig, Cantor Spectrum for the Almost Mathieu Operator, Comm. Math. Phys., 244(2), 297-309 (2004).

J. Mason, H. Handscomb, Chebyshev Polynomials, Chapman and Hall/CRC Press, 2003.

J. C. Abderramán, S. Sastre, E. Torrano Chebyshev Series in a 1-D Schrödinger Ergodic Operator. Proceedings of XX CEDYA - X CMA. Applied Math Spanish Congress. Sevilla, 2007. 